\title{
Estudio Multifactorial de Resistencia Última a Tensión Paralela a la Fibra en Muestras de Guadua angustifolia Kunth
}

\author{
Multifactorial Study of Last resistance to Parallel tension to the Fiber \\ in Samples of Guadua angustifolia Kunth
}

\author{
Caori Takeuchi T. ${ }^{1}$, Mauricio Duarte $\mathrm{T}^{2}$, John E. Alarcón G. ${ }^{3}$ y Jairo F. Olarte $\mathrm{F}^{4}$
}

\begin{abstract}
Resumen
Este artículo presenta la relación de la resistencia última a tensión paralela a la fibra de probetas de Guadua angustifolia procedentes de cuatro zonas del municipio de Pitalito-Huila, con factores como sección inferior, medio o superior y zona, determinada mediante un análisis de varianza multifactorial. La metodología para el corte de los culmos, obtención de las probetas y pruebas de resistencia a tensión, se basó en las normas NTC5300, NTC5525, NSR10 e ISO/TC 165N315, y en los protocolos para corte de culmos, probetas y determinación de la resistencia a tensión paralela a la fibra de la Universidad Nacional de Colombia sede Bogotá. Fueron ensayadas 120 probetas en la Universidad Nacional de Colombia sede Bogotá. El promedio de resistencia a la tensión paralela fue de 61,0 Mpa. El análisis multifactorial del diseño experimental, permitió identificar la significancia estadística de los factores sección y zona sobre la resistencia a la tensión con un valor-p inferior a 0,05 y un nivel de confianza del $95 \%$, la interacción de segundo orden no fue significativo.
\end{abstract}

Palabras clave: Guadua angustifolia; resistencia a la tensión paralela a la fibra; factores.

\section{Abstract}

This article presents the Parallel tension to the Fiber Strength in Samples of Guadua angustifolia specimens from four areas of Pitalito Huila in relation to factors such as base, intermediate or higher area, performed by multifactorial analysis of variance. The methodology for cutting the culms, obtaining test samples and tensile strength testing was based on the rules NTC5300, NTC5525, NSR10 and ISO / TC 165N315, and the cutting of culms protocols, test sample and determination of the tension parallel to the fibers strength of the Universidad Nacional de Colombia in Bogotá. 120 tests were performed at the Universidad Nacional de Colombia in Bogotá. The average value of the parallel tension strength was $61.0 \mathrm{MPa}$. Multivariate analysis of the experimental design, helped to identify the statistical significance of the section factors and area on the resistance to tension with a p-value less than 0.05 and a confidence level of $95 \%$, the second-order interaction was not significant.

Keywords: Angustifolia Guadua; resistance to parallel tension to the fibers, factors.

1 Magister en Estructuras. Docente Universidad Nacional de Colombia sede Bogotá. E-mail: cptakeuchit@unal.edu.co

2 Magister en Ingenieria Civil. Docente Universidad Surcolombiana. Av. Pastrana Borrero carrera $1^{2}$ Neiva. E-mail: maduto@ usco.educo

3 Ingeniero Agricola. Universidad Surcolombiana. Av. Pastrana Borrero carrera 1 Neiva. E-mail: rooney.guti@hotmail.com

4 Ingeniero Agricola. Universidad Surcolombiana. Av. Pastrana Borrero carrera $1^{\mathrm{N}}$ Neiva. E-mail: franchescoli82@hotmail.com

Recibido 30 de septiembre de 2013

Aprobado 29 de octubre de 2013 


\section{Introducción}

En Colombia, desde la época de la colonización, la guadua fue utilizada para la construcción de viviendas rurales y urbanas. Para los colonizadores antioqueños la guadua fue el símbolo de la civilización en dichas tierras. Este material también ha sido utilizado para la construcción de vivienda tradicional en los sectores menos favorecidos de la población (Garzón, 1996, citado por Gutiérrez et al., 2011).

Actualmente la Guadua angustifolia es considerada como alternativa ambiental y material estructural de grandes construcciones alrededor del mundo gracias a arquitectos como Simón Vélez que han motivado el interés de trabajar con materiales naturales como éste, contribuyendo al mejoramiento ambiental muy afectado por el uso de los materiales cotidianos en construcción.

En el municipio de Pitalito ubicado en el sur del departamento de Huila, se encuentra la especie Guadua angustifolia, la cual ha sido utilizada en su gran mayoría en el sector artesanal y constructivo. Las experiencias de sus pobladores debido a tradiciones familiares, han servido para la construcción de estructuras de tipo agricola como secadores solares, corrales, establos y beneficiaderos de café. La falta de conocimiento de las propiedades fisicas y mecánicas de la guadua en la región, y la mala información generada por algunos propietarios de los rodales que la consideran como maleza, hace que se ignore el verdadero aprovechamiento industrial de la especie como alternativa económica generadora de empleo e ingresos para las comunidades rurales del municipio de Pitalito-Huila.

Lo mencionado anteriormente, hace importante el estudio de las propiedades fisico-mecánicas de la guadua de este municipio y especificamente su comportamiento a tensión paralela a la fibra. Con la determinación del esfuerzo máximo a tensión paralela a la fibra, resulta apropiado analizar su comportamiento en relación con factores como procedencia de culmos y sección complementan- do investigaciones como las realizadas por López y Trujillo (2002), Janssen (2002), Castrillón \& Malaver (2004), Osorio \& Vélez (2005), Lozano et al. (2010), Gutiérrez (2011), donde se ha observado el incremento de la resistencia a tensión paralela con la altura del culmo de guadua.

\section{Metodología}

\subsection{Localización}

El material objeto de la investigación, provino del municipio de Pitalito al sur del departamento del Huila. En total se seleccionaron 4 rodales (zona de estudio A, B, C, D), y la selección de los sitios de muestreo se realizó de forma aleatoria. El rango de altitud de la zona de estudio fue de 1000 a 1800 m.s.n.m, con una temperatura media anual de 19 a $22^{\circ} \mathrm{C}$. Los límites de la zona de estudio, de acuerdo con el sistema de referencia de coordenadas MAGNA-SIRGAS fueron: Latitud $1^{\circ} 56,40.95 m \mathrm{~N}-1^{\circ} 50,26.30 \cdots \mathrm{N}$ y Longitud $76^{\circ} 57^{\prime} 15.00^{\prime \prime} \mathrm{O}-76^{\circ} 08,02.38 \cdots \mathrm{O}$.

\subsection{Etapas para el desarrollo del proyecto}

Para la selección, corte, caracterización física, determinación del comportamiento del esfuerzo a compresión paralela a la fibra, densidad básica y contenido de humedad, se trabajó con las siguientes normas, leyes y protocolos:

- Norma unificada para el manejo y aprovechamiento de la Guadua, (Carder, 2002).

- Ley forestal 1021 de 2006.

- ISO/TC $165 \mathrm{~N} 315,2001$. "Laboratory manual on testing methods for determination of physical and mechanical properties of bamboo"

- NTC5300 "Cosecha y postcosecha del culmo de Guadua angustifolia Kunth" (Icontec, 2008).

- NTC5525 "Métodos de ensayo para determinar las propiedades fisicas y mecánicas de la Guadua" (Incotec, 2007). 
- Reglamento colombiano de construcciones sismo-resistente NSR10, 2010. Titulo G "Estructuras de madera y estructuras de Guadua. Bogotá D.C

- Protocolos de la Universidad Nacional de Colombia sede Bogotá (2008). "Corte de culmos y probetas, determinación de la resistencia a tensión paralela a la fibra, medición del contenido de humedad para la Guadua angustifolia Kunth".

\subsubsection{Selección de culmos y Corte}

En esta etapa se seleccionaron e identificaron las guaduas maduras y se contó con la colaboración del personal del Tecnoparque 7 Agroecológico Yamboró del Servicio Nacional de Aprendizaje (SENA). Las guaduas, objeto de estudio, fueron cortadas a 1 metro por encima del cuello y posteriormente avinagradas (pérdida de humedad en la guadua) durante un periodo de 1 mes. Una vez terminado este proceso se cortaron en 3 secciones longitudinales: inferior $(1,5 \mathrm{~m})$, medio $(4,5 \mathrm{~m})$ y superior $(3,0 \mathrm{~m})$.

\subsubsection{Obtención de las probetas}

El material fue transportado en toletes de $50 \mathrm{~cm}$ de largo, al taller BIO-ARTEGUADUA ubicado en el municipio de Pitalito- Huila. Allí se realizó el trabajo de obtener las láminas o latas de $2,0 \mathrm{~cm}$ de ancho, de las cuales se escogieron 4 latas de cada tolete y fueron marcadas con sus respectivos caracteres el rodal, el número de la guadua, la sección de la guadua y una letra que identifica cada lata (a, b, c y d); posteriormente las latas se trasladaron hacia la ciudad de Neiva, donde se seleccionaron dos latas de las cuatro disponibles de cada tolete, a las cuales se les realizó un trazado mediante una plantilla con la forma y las dimensiones deseadas para la probeta. Luego de este proceso se elaboraron 288 probetas con una sierra sinfin. Posteriormente, estas probetas se enviaron a la Universidad Nacional de Colombia sede Bogotá donde se tomaron las medidas de ancho y espesor para determinar el área de falla.

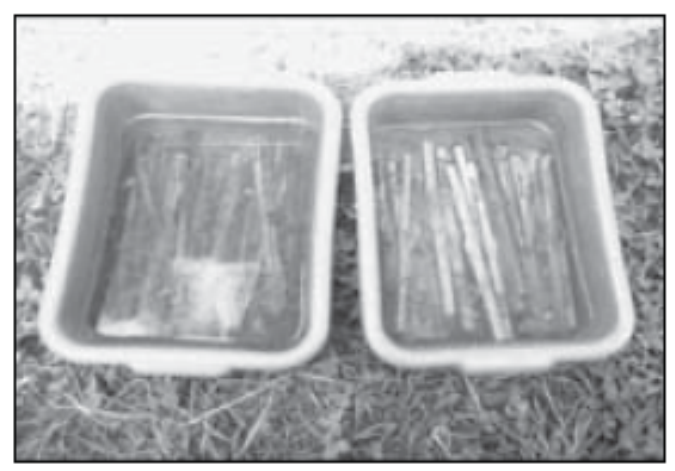

Figura 1. Inmersión en agua de las probetas

Una vez medidas, estas probetas fueron sumergirlas en agua durante un periodo aproximado de 2 semanas, para evitar el fisuramiento por pérdida de humedad y obtener valores conservadores de resistencia (figura 1).

\subsubsection{Ensayos de resistencia a la Tensión para- lela a la fibra}

Los ensayos se realizaron, con el equipo de ensayos marca VERSA TESTER (figura 2), con capacidad de carga de 30 Toneladas. Del total de las probetas realizadas, se ensayaron 178 , de las cuales fueron descartadas 58 por presentar una falla diferente a la esperada ya fuera por corte,

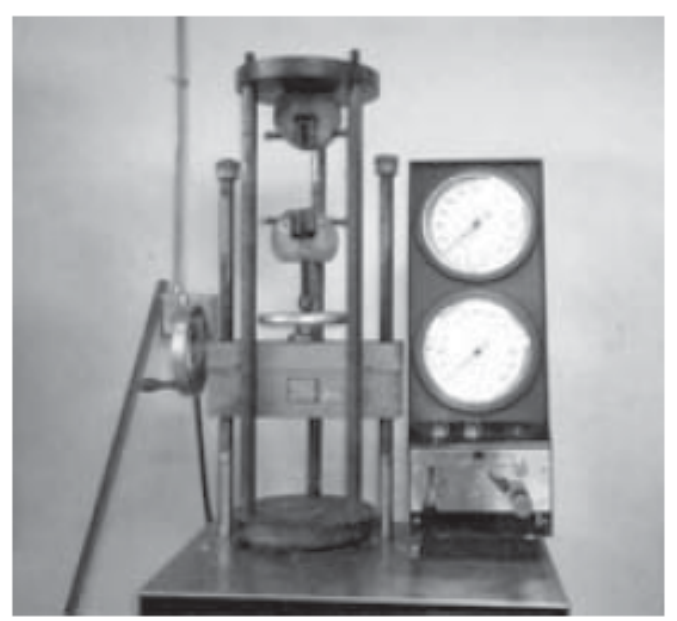

Figura 2. Equipo de ensayo a tensión paralela. 
por punzonamiento o por daños producidos por las mordazas. La velocidad de aplicación de carga fue de de $0,01 \mathrm{~mm} / \mathrm{s}$ aproximadamente. Una vez finalizados los ensayos de resistencia a la tensión paralela a la fibra, se procedió a determinar el contenido de humedad para cada probeta; para este ensayo se extrajo una lámina de la probeta para luego ser introducidas al horno de secado a una temperatura de $103^{\circ} \mathrm{C} \pm 2^{\circ} \mathrm{C}$, como lo establece la Norma Técnica Colombiana NTC 5525.

\subsubsection{Análisis estadistico (ANOVA MULTI- FACTOR)}

Para el análisis estadístico se utilizó un diseño de varianza multifactorial totalmente aleatorio, donde se evaluaron las resistencias a tensión paralela a la fibra de las probetas ensayadas. Previamente se descartaron los valores atipicos mediante análisis exploratorio; la normalidad se verificó mediante la prueba de Shapiro-Wilk. La variable respuesta para el diseño ANOVA multifactorial, los factores y niveles de factor se muestran en la tabla 1.

Tabla 1. Factores y niveles de factor cuyos efectos sobre la resistencia a la compresión fueron evaluados.

\begin{tabular}{|c|c|c|}
\hline Factor & Niveles de factor & $\begin{array}{l}\text { Variable } \\
\text { dependiente }\end{array}$ \\
\hline \multirow{4}{*}{ 1.Rodal } & 1.La Esperanza & \multirow{7}{*}{$\begin{array}{l}\text { Esfuerzo a la Ten- } \\
\text { sión paralela a la } \\
\text { fibra (Mpa) }\end{array}$} \\
\hline & 2. Villa María & \\
\hline & 3. La Dalia & \\
\hline & 4. Yamboró & \\
\hline \multirow{3}{*}{ 2. Sección } & 1. Inferior & \\
\hline & 2. Medio & \\
\hline & 3. Superior & \\
\hline
\end{tabular}

El nivel de confiabilidad minimo para los resultados fue del 95\%, los datos se organizaron y se procesaron en el software STATGRAPHICS CENTURION versión 15.2.06 asumiendo como hipótesis la normalidad, homocedasticidad e independencia de los mismos.

\section{Resultados}

Las probetas ensayadas a tensión paralela a la fibra, presentaron una densidad anhidrida promedio de $687,08 \mathrm{~kg} / \mathrm{m}^{3}$ con una desviación estándar de $53,90 \mathrm{~kg} / \mathrm{m}^{3}$ y contenidos de humedad promedio de $101,25 \%$ con una desviación estándar de $11,91 \%$. Se analizaron 115 probetas en el ANOVA multifactorial, la aleatoriedad en el muestreo, obtención de probetas y ejecución de ensayos, garantizaron la normalidad, independencia y heterocedasticidad de los residuos.

\subsection{ANOVA Multifactorial}

El análisis de varianza multifactorial presentado en la tabla 2, permitió identificar la significancia estadística del factor sección y zona sobre la resistencia a la tensión paralela a la fibra con un valor-p inferior a 0,05 y un nivel de confianza del 95\%; las interacciones de segundo grado no produjeron efectos significativos sobre la resistencia de la guadua.

Tabla 2. Análisis de varianza multifactorial para resistencia a la tensión paralela a la fibra.

\begin{tabular}{lccccc}
\hline Fuente & $\begin{array}{c}\text { Suma } \\
\text { de } \\
\text { Cua- } \\
\text { drados }\end{array}$ & Gl & $\begin{array}{c}\text { Cua- } \\
\text { drado } \\
\text { Medio }\end{array}$ & $\begin{array}{c}\text { Ra- } \\
\text { zón- } \\
\text { F }\end{array}$ & $\begin{array}{c}\text { Valor } \\
\text { P }\end{array}$ \\
\hline $\begin{array}{l}\text { Efectos } \\
\text { Principales }\end{array}$ & & & & & \\
\hline A:seccion & 229,07 & 2,00 & 114,54 & 3,33 & 0,04 \\
\hline B:zona & 401,96 & 3,00 & 133,99 & 3,90 & 0,01 \\
\hline Interacciones & & & & & \\
\hline AB & 122,80 & 6,00 & 20,467 & 0,60 & 0,73 \\
\hline Residuos & 3539,90 & 103,00 & 34,37 & & \\
\hline $\begin{array}{l}\text { Total } \\
\text { (Corregido) }\end{array}$ & 4292,59 & 114,00 & & & \\
\hline
\end{tabular}

\subsection{Pruebas de múltiples rangos (Efectos prin- cipales)}

La comparación múltiple entre los niveles del factor zona que se presenta en la tabla 3, demues- 
tra diferencias significativas entre la zona B y las demás, con el valor más bajo de resistencia promedio. Se evidencia homogeneidad entre la resistencia de la guadua de las zonas A, C y D, lo que significaría condiciones similares de sitio.

Tabla 3. Prueba de múltiples rangos para resistencia a la compresión por Zona.

\begin{tabular}{|c|c|c|c|c|c|}
\hline \multirow{2}{*}{$\begin{array}{l}\text { Datos } \\
\text { Estadísticos }\end{array}$} & \multicolumn{4}{|c|}{ Zonas } & \multirow{2}{*}{ Total } \\
\hline & A & B & $\mathrm{C}$ & D & \\
\hline Casos & 26,00 & 30,00 & 29,00 & 30,00 & 115,00 \\
\hline Media (Mpa) & $62,64^{Q}$ & $57,95^{p}$ & $61,26^{Q}$ & $62,31^{Q}$ & 61,00 \\
\hline $\begin{array}{l}\text { Desviación } \\
\text { Estándar } \\
\text { (Mpa) }\end{array}$ & 7,10 & 4,67 & 6,49 & 5,26 & 6,13 \\
\hline $\begin{array}{l}\text { Coeficiente de } \\
\text { variación (\%) }\end{array}$ & 11,33 & 8,07 & 10,60 & 8,45 & 10,05 \\
\hline Mínimo (Mpa) & 49,81 & 51,56 & 48,71 & 51,18 & 48,71 \\
\hline Máximo (Mpa) & 75,15 & 67,01 & 75,00 & 74,00 & 75,15 \\
\hline Limite Inferior & 60,36 & 55,83 & 59,10 & 60,19 & 55,83 \\
\hline $\begin{array}{l}\text { Limite Supe- } \\
\text { rior }\end{array}$ & 64,93 & 60,08 & 63,43 & 64,43 & 64,93 \\
\hline
\end{tabular}

Se encontró que la sección del culmo de la Guadua angustifolia es significativa sobre la resistencia a la tensión paralela a la fibra de acuerdo con el análisis de varianza. La parte superior es la más resistente y difiere significativamente de los valores promedio de las secciones media e inferior; tal como se indica en la tabla 4. Se confirma lo indicado por investigadores como Gutiérrez (2011), López \& Correal (2009), Pacheco (2006), Uribe \& Durán (2002), entre otros; que han descrito que la resistencia de los culmos a tensión es mayor en la sección superior disminuyendo hacia la sección inferior.

Otros estudios de algunos autores, han encontrado diferentes valores para referirse a la resistencia última a tensión en probetas de guadua. Por ejemplo, Gutiérrez (2011) encontró un valor promedio de esfuerzo último de $86.96 \mathrm{MPa}$. Lozano et al. (2010) hallaron un valor de esfuerzo último promedio de $61.40 \mathrm{MPa}$. Ciro, Osorio \& Vélez (2005) encontraron que el valor promedio de es- fuerzo último promedio fue de $190.70 \mathrm{MPa}$. Por otra parte Castrillón \& Malaver (2004) de los ensayos realizados obtuvieron un esfuerzo último promedio de $91.87 \mathrm{MPa}$. Del mismo modo Janssen (2002) presenta un valor promedio de 148.40 $\mathrm{MPa}$. Siguiendo las normas del INBAR, López y Trujillo (2002) hallaron un valor de esfuerzo ultimo promedio de $53.5 \mathrm{MPa}$.

Tabla 4. Prueba de múltiple rangos para resistencia a la tensión por Sección.

\begin{tabular}{lcccc}
\hline \multirow{1}{*}{$\begin{array}{c}\text { Datos } \\
\text { Estadisticos }\end{array}$} & \multicolumn{4}{c}{ Sección } \\
\cline { 2 - 5 } & Inferior & Media & Superior & Total \\
\hline Casos & 38,00 & 38,00 & 39,00 & 115,00 \\
\hline Media (Mpa) & $60,77^{\mathrm{Q}}$ & $59,46^{\mathrm{Q}}$ & $62,89^{\mathrm{p}}$ & 61,00 \\
\hline $\begin{array}{l}\text { Desviación } \\
\text { Estándar (Mpa) }\end{array}$ & 5,95 & 7,13 & 4,79 & 6,13 \\
\hline $\begin{array}{l}\text { Coeficiente de } \\
\text { variación (\%) }\end{array}$ & 9,79 & 12,00 & 7,63 & 10,05 \\
\hline Minimo (Mpa) & 50,47 & 48,71 & 53,75 & 48,71 \\
\hline Máximo (Mpa) & 75,00 & 75,15 & 72,77 & 75,15 \\
\hline Limite Inferior & 58,89 & 57,57 & 61,03 & 55,83 \\
\hline Limite Superior & 62,66 & 61,36 & 64,75 & 64,93 \\
\hline $\begin{array}{l}\text { Letras differentes para la media Indican dlffrenclas estadisficamente slg- } \\
\text { nlficatlvas con un nlvel de confianza del } 95 \% .\end{array}$ & &
\end{tabular}

\section{Conclusiones}

El factor zona es significativo sobre la variable respuesta resistencia a la tensión paralela a la fibra, con un valor-p de 0,01 . Solo se encontró para la zona B diferencias minimas significativas en comparación con las demás zonas de estudio según lo establecido por la prueba LSD de Fisher.

La sección del culmo es un factor significativo sobre la resistencia a la tensión con valor-p de 0,04 . Se encontraron diferencias estadisticamente significativas entre valores de resistencia promedio de las secciones inferior y media frente al valor obtenido por la sección superior. Los valores promedio para las cuatro zonas para las secciones inferior y media fueron de: 60,77 y 59,46 Mpa respectivamente; valores que se encuentran por debajo de la resistencia alcanzada para la sección superior $62,89 \mathrm{Mpa}$. 
Los efectos de las interacciones entre factores no son significativos. El valor-p para la interacción entre zona y sección fue de 0,73 .

\section{Referencias Bibliográficas}

1. Castrillón, B., Malaver, D., 2004. Procedimientos de ensayo para la determinación de las propiedades fisico mecánicas de la guadua. Tesis de grado. Bogotá: Universidad Nacional de Colombia. 94 p.

2. Ciro. V., Jairo A., Vélez, R., Juan y OSORIO, S., 2005. Determinación de la resistencia mecánica a tensión y cizalladura de la Guadua Angustifolia Kunth. Medellin: Rev.Fac.Nal.Agr. V.58, No 1.p. 2709-2715

3. Garzón, C., Jenny. V., 1996. Optimización de uniones en guadua: Trabajo estructural de uniones a tensión. Universidad Nacional de Colombia

4. Giraldo, E., Sabogal, A., 2002. La GUADUA una alternativa sostenible. Publicación de la corporación autónoma regional del Quindio, CRQ. Quindio, 1999. 42 p. Citado por: URIBE, Maritza y DURÁN, Alejandro. Estudio de elementos solicitados a compresión armados por tres guaduas. Tesis de grado. Bogotá: Universidad Nacional de Colombia. Departamento de Ingeniería Civil. $125 \mathrm{p}$

5. Icontec, Instituto colombiano de normas técnicas, 2007. Métodos de ensayo para determinar las propiedades fisicas y mecánicas de la Guadua angustifolia Kunth. NTC 5525. Bogotá D.C

6. Icontec, Instituto colombiano de normas técnicas, 2008. Cosecha y postcosecha del culmo de Guadua angustifolia Kunth. NTC 5300. Bogotá D.C
7. Lozano, J., Luna, Patricia., Takeuchi, Caori., 2010. Validación de la Guadua angustifolia como material estructural para diseño, por el método de esfuerzos admisibles. Bogotá: Universidad Nacional de Colombia. $190 \mathrm{pp}$

8. López, L., Trujillo, D., 2002.Diseño de uniones y elementos en estructuras de guadua. Pereira: Seminario - Taller Avances en la investigación sobre guadua.

9. Reglamento Colombiano de Construcciones Sismo-resistente NSR10, 2010. Titulo G "Estructuras de madera y estructuras de Guadua. Bogotá D.C.

10. Pacheco, C., 2006. Resistencia a la tracción perpendicular a la fibra de la guadua angustifolia. Trabajo de grado. Bogotá. Universidad Nacional de Colombia. Departamento de Ingeniería Civil y Agricola. $132 \mathrm{p}$.

11. The international organization for standardization, 2001. Laboratory manual on testing methods for determination of physical and mechanical properties of bamboo. ISO/TC $165 \mathrm{~N} 315$

12. Universidad Nacional de Colombia, 2008. Protocolos de la Universidad Nacional de Colombia sede Bogotá, Corte de culmos y probetas, determinación de la resistencia a tensión paralela a la fibra, medición del contenido de humedad para la Guadua angustifolia Kunth. Bogotá

13. Uribe, M., Durán, A., 2002. Estudio de elementos solicitados a compresión armados por tres Guaduas. Bogotá: Universidad Nacional de Colombia. Departamento de Ingeniería Civil. 125 pp. 